Research Article

\title{
Design and Implementation of Multidimensional Interaction in Online English Course under the Assistance of Omnimedia
}

\author{
Ming Cao \\ School of Humanities and International Education, Xi'an Peihua University, Xi'an, China \\ Correspondence should be addressed to Ming Cao; caoming@peihua.edu.cn
}

Received 29 July 2021; Accepted 6 September 2021; Published 18 September 2021

Academic Editor: Dinesh Kumar Saini

Copyright ( 92021 Ming Cao. This is an open access article distributed under the Creative Commons Attribution License, which permits unrestricted use, distribution, and reproduction in any medium, provided the original work is properly cited.

\begin{abstract}
The method of multidimensional interaction in online teaching and learning requires teachers to harness the techniques of omnimedia and have an overall course design to achieve similar results as offline learning. Supported by QQ, Lanmo Cloud Class, and WeChat platforms, online English courses implement multidimensional interaction method to enhance language communication. This method compensates for the inefficacy of online teaching and learning which falls short of face-to-face interaction and considers the needs of students. The quasiexperimental study sampled 66 freshmen English major students in an ordinary college via Integrated English Online Course for three months. By using T-test of SPSS 26.0, statistics showed that there were significant differences between the experimental class (Number 35) and the control class (Number 31) in students' feedback $(p=0.044,<0.05)$ and the results of their final examinations $(p=0.049,<0.05)$. The results indicated that multidimensional interaction in online English course under the assistance of omnimedia appeared effective. It also reflected that both teachers and students should grasp the skills of using new technology and make good use of omnimedia network platform. Teachers need to change traditional teaching concept to student-centered and create effective teaching design while students need to overcome the discomfort and actively engage in online learning to achieve the goals.
\end{abstract}

\section{Introduction}

The rapid development of science and technology has provided unlimited possibilities for online teaching and learning. The emergence of new technologies and concepts such as "Internet Plus," "Cloud Computing," and "Big Data," for example, has made online learning more flexible and convenient. New technologies have provided frontline teachers with the resources and opportunities to integrate new technology into English teaching. Liu et al. reported that, in the "Internet Plus" environment supported by Adobe Connect platform, Sino-US cross-culture EFL (English as a foreign language) blended teaching class with real-time technology made a positive impact on the Chinese students' learning behaviours, increased their self-efficacy, and enhanced their understanding of the target culture. At the same time, the unique and rich learning resources and new perspectives they brought into the cyber class benefited students in both countries [1]. "Cloud Computing" is a model of paying by the amount of data using and follows the learning principle of 5A (Anyone, Anytime, Anywhere, Any device, Any form). Yang et al. created a model of smart learning environment based on Cloud Computing. It has been noticed to be of great significance to carry out related research on Cloud Computing and smart learning environment [2]. Although there is less empirical research on the EFL field, some scholars tried to use "Cloud Computing" perspective into college teaching and learning for translation major [3]. In terms of "Big Data," some believe that it is a field that an overlarge and hypercomplex dataset is provided for traditional data system application software to analyze, withdraw, or manage [4]. Others consider that it is so large, fast, or complex that it is difficult or impossible to process using traditional methods [5]. In EFL field, researchers explored many ways of using "Big Data" to design their teaching and learning. It is verified that, by using Google images to be a dataset, students created pictorial annotations for them to remember the target words and integrated them 
into their prior knowledge [6]. In "Big Data" related English teaching, students' learning motivation level and learning ability have increased by $16.6 \%$ and $14 \%$, respectively $[7,8]$. As a result, these physical changes flourish the way of integrating technology into English teaching and learning.

In addition, it has allowed for the change from teachercentered to student-centered methods, bringing studentcentered instruction into practice. Despite the variety of research focused on online teaching and learning in recent years $[9,10]$, with some spotlight on online interaction strategies [11], there are only a small number of studies on the topic of implementing online teaching with effective interaction. In particular, there has been a lack of attention on the relationship between high quality online teaching at local colleges and the technologies that support the teacherstudent interactions. It is reported that teachers' adaptation is always required to find and solve problems during the teaching practice in an online and offline blended program. Students changed the attitudes gradually under the help of teachers and gain improvement in writing quality [12]. Interaction plays the key role when teachers carry out student-centered instruction. Thus, we need more cases to show how technology integrates into English teaching and learning. Along with the normalization of online or a combination of online-offline teaching and learning, implementing effective Internet-based teaching has become a new challenge for college teachers. This challenge raises some questions: how are we going to draw and maintain students' attention to make teaching and learning more effective? What do students think of Internet-based learning in central provinces of Chinese mainland? How does online learning impact student results? Interaction strategies have become the key of Internet-based teaching and learning. To achieve student-centered online learning results, we use multidimensional interaction strategies with the assistance of omnimedia in designing the course. New thoughts and ideas may arise during implementation.

\subsection{Relevant Concepts}

1.1.1. Interaction and Multidimensional Interaction. Interaction is a back and forth activity between subjects and objects. It is a form of communication [13]. Ancient Chinese ideologies include interaction when teaching as a key part of education. For example, during the Warring States Period of ancient China, in Xueji of The Book of Rites, it included "question-answer" and "analysis-explanation" teaching strategies. While teaching, the teacher should not repeat what the book said without first considering what the student knew. Teachers should not give answers until students had no more questions to ask [14]. In 1989, Moore classified interaction and this classification included three types of interaction: student-teacher interaction, student-student interaction, and student-content interaction [15]. Teacherstudent interaction is based on the teacher's hypothesis of how much of the material the students have grasped. Student-student interaction is based on the concept of equality in which students feel free to discuss concepts and what they have understood with their peers when teachers and authority figures are absent. Student-content interaction has been understood to be a unique interaction between students and the content they are learning, including how students understand the learning materials, the concepts from material, and the cognitive structure in students' minds. This dialogue may also be called "an internal monologue" [15]. Multidimensional interaction stemmed from the context of interaction. Palinscar and Brown put forward the concept of multidimensional interaction in the 1970s last century under the concept of constructivism. They proposed that multidimensional interaction method required teachers to keep the idea of student-centered learning while teaching. Teachers should enable students to shift from passively receiving information to actively and autonomously pursuing information [16]. Evidently, multidimensional interaction method places the emphasis on student-centered learning; by fully understanding the concept of interaction, teachers can utilize multidimensional interaction method to effectively design courses for their students.

Scholars often use online conference, online case analysis, video playback, and virtual activities to achieve multidimensional interaction. They have tried to overcome the limitations of the online learning facilities and optimize online interaction. In addition, they have implemented the multidimensional activities to make student-student, teacher-student, and student-content types of interaction apply in multiangle, multiaspect, and multilevel [17]. Other scholars believe that implementation of multidimensional online teaching or hybrid of online and offline teaching needs teachers to analyze many factors such as students, course contents, students' learning strategies, and learning environment until we reach the ideal result [10]. If we use different Internet platforms effectively, we may achieve close teacher-student relationship [18]. However, some scholars hold a cautious attitude in how they regard online interaction, as they believe the positive influence for learners is limited to the period of online interaction for the second language vocabulary learning [19]. Consequently, in order to realize online teaching and learning under the assistance of omnimedia and achieve similar results as offline learning, teachers need to not only be familiar with the different online platforms, but also be well-versed about how to design their online course and pay attention to their students' feedback and feelings. Multidimensional interaction presents an inevitable question to both teachers and students. If we wisely use computer and mobile phones, taking care of those interaction, we will arouse the enthusiasm of students' motivation, pay attention to learning process, and promote student-centered online teaching and learning.

1.1.2. Omnimedia and English Teaching. There are different explanations to omnimedia. Most of them tend to explain that, by using different media (platform), combining different ways of spreading, and converting or transplanting signals, people have more ways of acquiring information [20]. These ideas assist learners in achieving learning in different places with methods that are less costly and more 
profitable. The features can be explained using pictures, letters, sounds, light, images, etc., to send messages in certain platforms. Learners may also use computers, mobile phones, tablets, and smart TV sets to receive messages. Hence, everyone can access the learning systems at any time and place by using available terminals to receive the learning content they want and to fully utilize the service [21]. Based on the statistics from the Ministry of Education of People's Republic of China of 2018, China has 2.76 hundred million students in all forms of education at all levels [22]. If we are able to use the omnimedia resources to serve those students, it will provide an opportunity to have a meaningful exploration while combining information technology with education.

Language learning has its own special requirements. The expression of teachers and the practice of students are highly related to interaction. In offline in-person learning, interaction can be all-rounded with the teacher's voice and gestures, including eye contact, facial expression, hand signals, posture, and so forth. These all-rounded expressions with rich emotions are signals to draw students' attention easily. In case that the student is slightly distracted, they may miss the teacher's instruction but still be able to interpret the meaning from the teacher's gestures and expressions. Online learning is a unique situation in which the teachers and students are physically separated making it difficult to fully interact with one another. However, different kinds of Internet platforms provide us another chance to manage this shortcoming. By effectively utilizing pictures, letters, characters, sounds, and images, etc., teachers and students can exchange information attentively and even avoid some other irrelevant distractions to realize a state of highly focused teaching.

1.2. Design. It is not rare to integrate new technology into teaching and learning design in recent years. The common platforms include Facebook, Moodle, Google, Adobe Connect, Tencent, Lanmo Cloud Class, and so forth. The educational potential and advantages have been compared between Facebook and Moodle platforms, Tencent and Lanmo Cloud Class, and others [23, 24]. Currently, many teachers try to use different platforms at the same time to offset the shortage and promote the advantages. To realize multidimensional interaction, English online teaching and learning have mainly relied on QQ, Lanmo Cloud Class, and WeChat platforms. It is called omnimedia as it combines different ways of spreading and converting or transplanting signals. No matter what platform it is, the supporting system such as hardware, software, and Internet also plays an important role. The design can be seen as shown in Figure 1.

1.3. Implementation. QQ platform is one of the application products from Tencent Company. It is user friendly and is also the most frequently used platform. Both teachers and students can use it to perform many tasks such as lecturing, group discussion, and conference, for example. It gives a chance for students to set up their own discussions without instructor interference. Lanmo Cloud Class has many functions such as brainstorming, first-to-answer, light live streaming, messaging, discussion, group discussion, voting, questionnaire, reporting, displaying, debating, and testing. In reality, QQ and Lanmo are synergistically providing support to students. WeChat platform is also from Tencent and is flexible and easy to be handled for teacher-student interaction. It supplements the other two formal platforms as students often communicate with teachers on WeChat platform outside of class.

1.3.1. QQ Platform. QQ platform is the most commonly used platform. It can be used as lecturing, conference, and discussion with multiple interactive functions. It is quite similar to the offline classroom loved by teachers and students. In addition, it contains a mentoring function, which strengthens teacher-student relationship. Students often use this platform to start discussions and complete group assignments. Here are some examples of the QQ platform application (Figure 2).

1.3.2. Lanmo Cloud Class Platform. There are many functions in Lanmo Cloud Class. Teachers use the platform to supplement lectures presented on QQ platform. During the lecture of traditional teaching, teachers often talk by themselves without giving the opportunity to communicate with students. While with student-centered presenting, teachers can cast questions using the Lanmo platform and receive responses immediately. Depending on the content of the lecture, teachers either set up questions for the language learning or explore the supporting information. On the other terminal, students can quickly pick the questions, express their own ideas, and discuss with their peers. When they become familiar with the platform, they will be able to fully take advantage of the available applications. Not only do they learn the technology, but students also develop their thinking, expand their vocabulary, and practice their language skills. Some typical interaction applications are shown as follows:

(1) Brainstorming block: it uses letters, words, and symbols to stimulate interaction. When the teacher posts a question, students can quickly cast their ideas, thoughts, or answers. This mimics offline class in which students present their own ideas to solve the problems and inspire their peers to contribute as well. The brain storming record is shown in Figure 3.

(2) First-to-answer block: it is a good way to draw students' attentions. First, when a question is posted on the screen, students can click the icon to grab the chance to answer. This is called the "raise-yourhand" function. If the question is too difficult, the teacher will give certain clues or delete an option to lower the difficulty. Teachers can also use a specific function to choose one student to answer the question randomly, which is called the "shake-yourphone" function. While using the first function, it is the students who determine the results and in the second available function, it is the teacher who determines the results. Here are screenshots of first-toanswer block (Figure 4) 


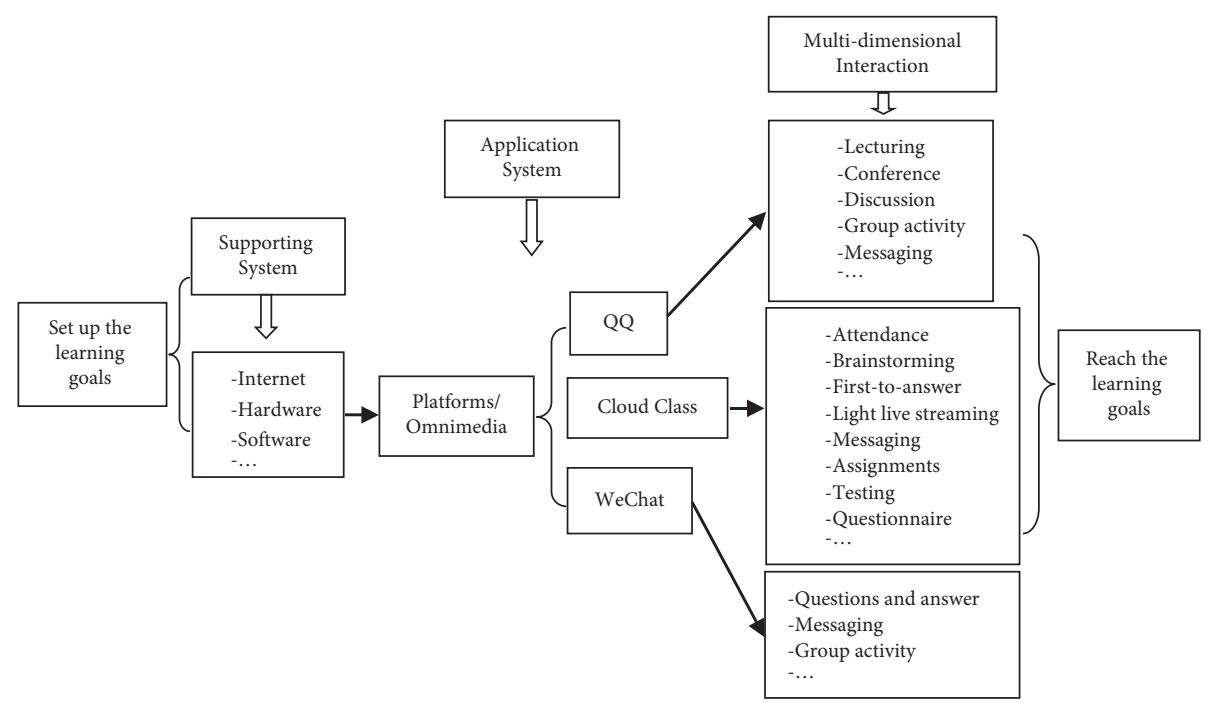

FIgURE 1: Multidimensional interaction design of online learning.
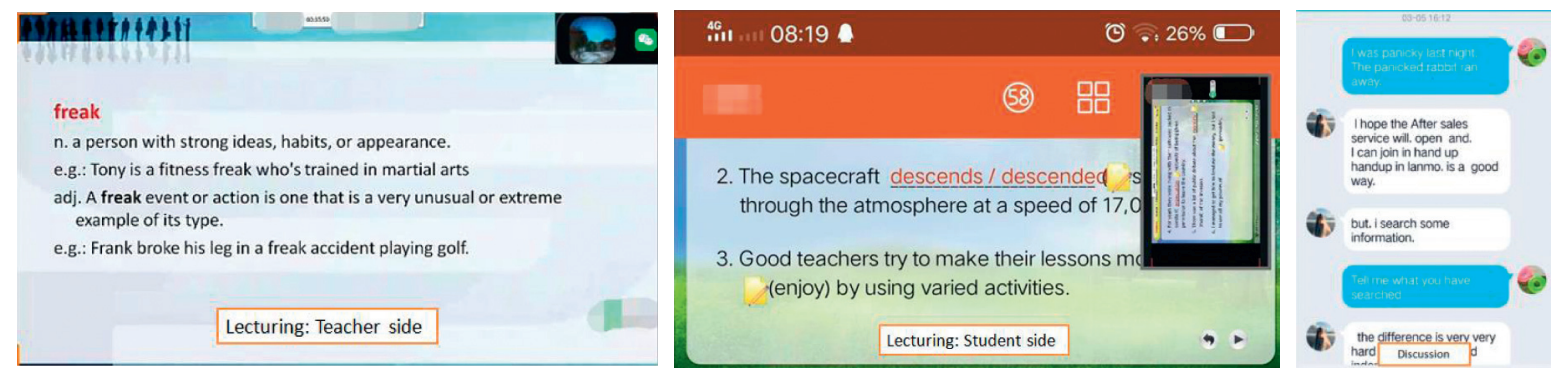

FIGURE 2: Screenshot of QQ platform interaction.
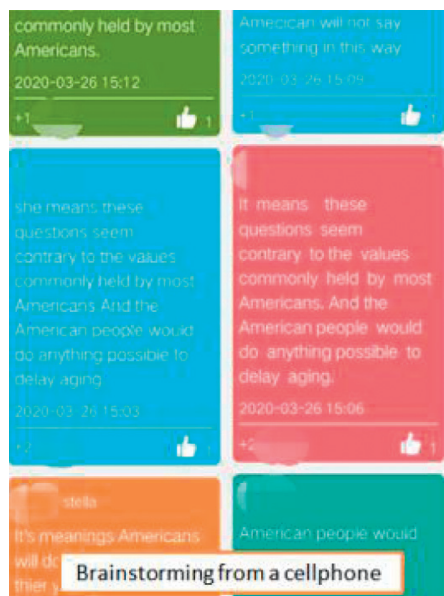

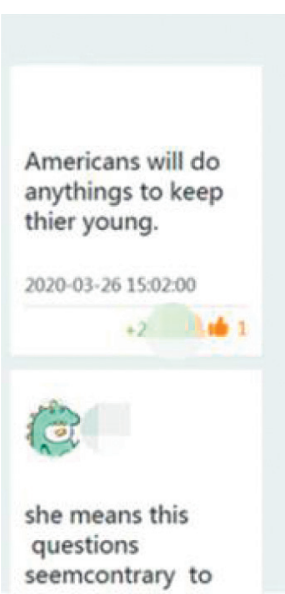

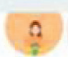

It means these questions seem contrary to the values commonly held by most Americans. And the American people would do anything possible to delay aging.

2020-03-26 15:06:44

\section{Brainstorming from a computer}

Brainstorm of Unit 2

contrary to the values commonly held by most Americans.And the American people would do anything possible to delay aging.

2020-03-26 15-03:45 +2 แh 1

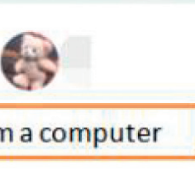

It's not regular spoken English in America.

2020-03-26 14:57:24
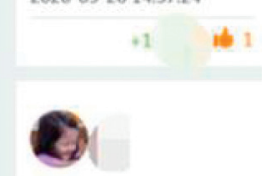

It means in American people will do anything to keep young.

2020-03-26 14:52:05

Figure 3: Screenshot of brainstorming on cellphone and computer screen.

(3) Light live streaming block: it, similar to live audio clips, allows teachers to provide short explanations of the course content to students, which lightens their study load and assists their understanding. Teachers are able to explain bit by bit until the students understand. These explanations can take the form of sound message, pictures, or videos. Typed text is seldom used because it is too slow. It can also be used as a discussion forum allowing students to post their ideas in tandem with the explanations. This is a very effective way to involve students in the discussion. Technically, it saves Internet flow of data. Screenshot is as shown in Figure 5. 


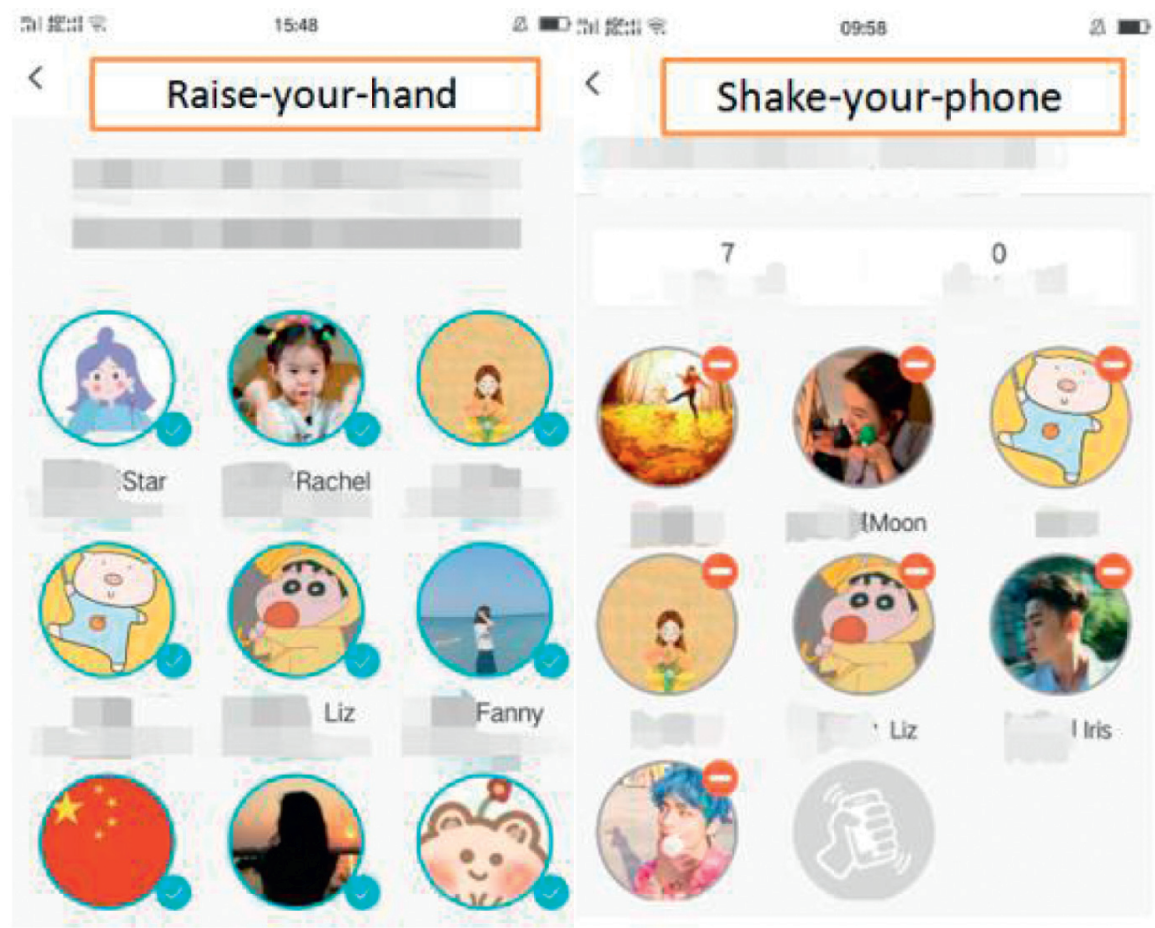

FIGURE 4: Screenshot of "first-to-answer" function.

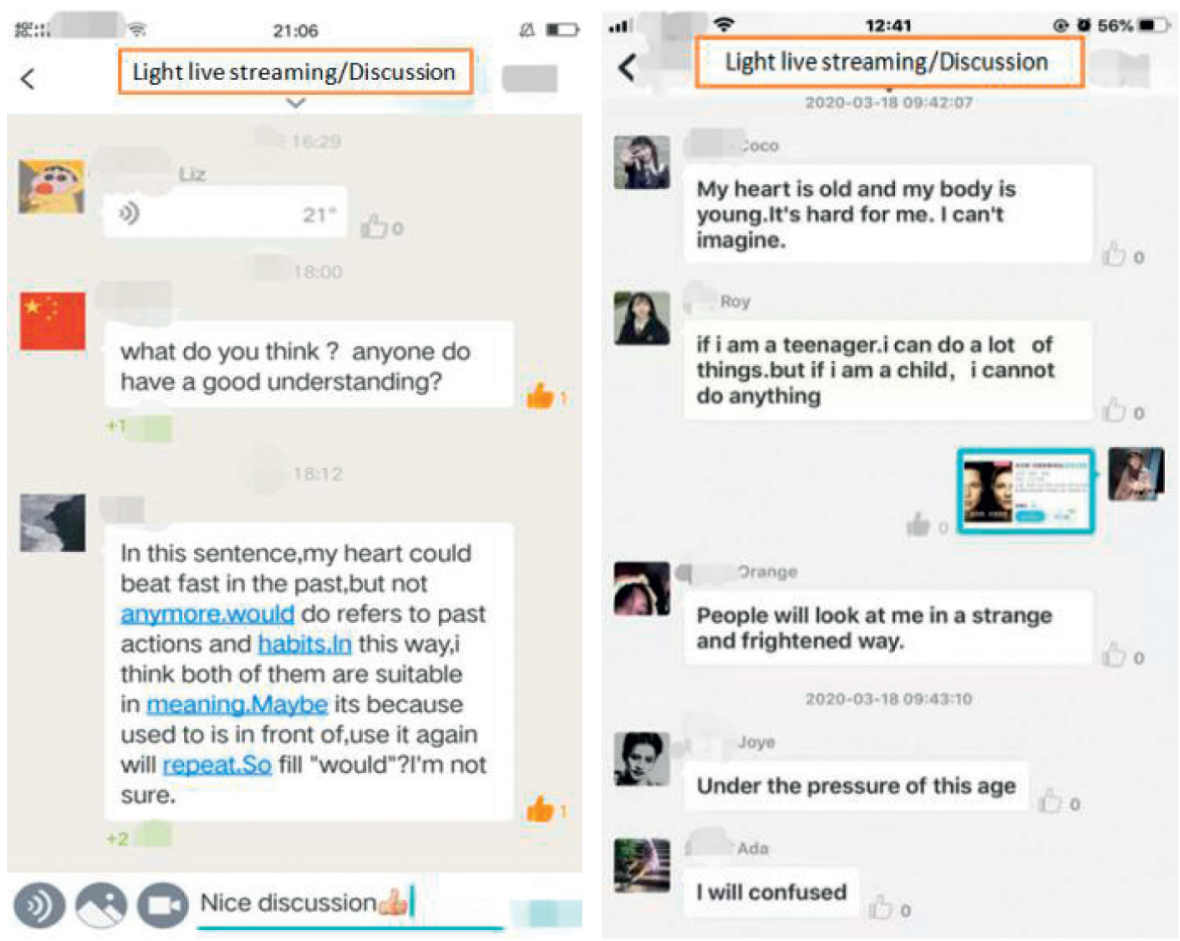

FIGURE 5: Screenshot of light live streaming and discussion.

(4) Messaging block. it is another way to interact and is mainly used outside of class. Teachers can post messages in the online bulletin board and inform or remind students when necessary. Words and text are most frequently used in this form of interaction and the important points can be also highlighted. Here are some examples of messaging block (Figure 6). 


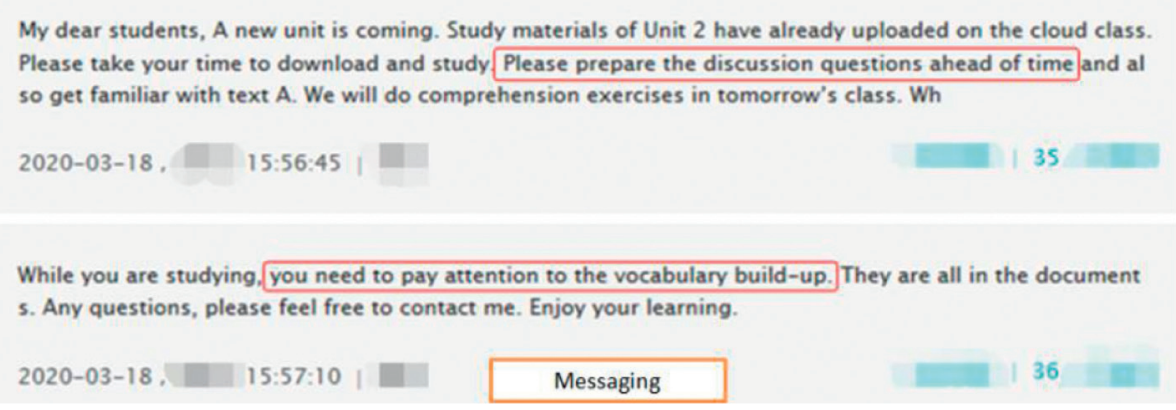

FIgURE 6: Screenshot of messaging block.

1.3.3. WeChat Platform. WeChat plays an important role as another form of connection between teachers and students outside of class. Not only is it easy to access, but it also allows students to ask questions or leave messages when needed. Moreover, the various lively formats make students and teachers express ideas quickly and effectively. The platform provides letters, characters, words, sound, symbols, audio, and videos and other mixed formats to enhance communication. Here is the WeChat screenshot (Figure 7).

Above are some typical multidimensional interaction examples from QQ, Lanmo Cloud Class, and WeChat platform. There are also other functions that aid interaction. Without any interruptions, words, pictures, sounds, audios, and videos are the main forms of the three-type interactions. It improves participation of teachers and students and embodies the superiority of omnimedia in assistance of online English teaching and learning.

1.4. Quasiexperiment Research. In order to understand and investigate students' feelings and attitudes toward online multidimensional interaction under the assistance of omnimedia, as well as its influence on online English teaching and learning, a self-made questionnaire has been designed. At the same time, we compared the results of students' final examination as a reference point. The questionnaire has been released by one of the most popular online platforms in China, Wen Juan Xing, and the data has been processed by SPSS 26.0.

1.4.1. Investigation of Questionnaire. 66 freshmen English major students have been sampled by random in an ordinary college of a central province in Chinese mainland. These students are English majors taking Integrated English Online Course during research. Multidimensional interaction under the assistance of omnimedia has been applied for experimental group (No. 36) and traditional method under the assistance of single platform for control group (No. 31). The students' score of College Entrance Examination has no significant differences. They were not aware of the experiment. The questionnaire has been designed by Likert classification of five levels with 16 questions in total. The collection rate of the questionnaire for the two groups was $97.22 \%$ and $100 \%$, respectively. The 16 questions with responses are shown in Table 1.
The data above have been analyzed by the software of SPSS 26.0. T-test showed that, within the answers "completely agree" and "agree," experimental group (No. 35) has significant differences $(p=0.44,<0.05)$ compared with the control group (No. 31). It indicated that multidimensional interaction brought different feelings and attitudes toward students' online study. Experimental students showed more acceptable attitudes toward this method. Students who held the neutral attitudes also have significant difference $(p=0.000,<0.001)$, which means the control group students had more tendency to not care about the interaction or had no sense about interaction in online course. There were no significant differences between two groups in answering "disagree" and "completely disagree." It showed that very few students do not have influences despite the multidimensional interaction mode or traditional mode of online learning (Table 2).

Taking some points as examples, $74.29 \%$ experimental group students expressed that there were no big differences but $45.16 \%$ expressed that it had differences; $68.57 \%$ experimental students believed that, in the online class of multidimensional interaction mode, students can get more chances to open their mouth, whereas only $48.39 \%$ control group students feel they had chances to speak out; regarding "shake-your phone" and "raise-your-hand" of interaction, nearly $71.43 \%$ experimental group students expressed that they love the portion as they felt like involved in the group, while $54.84 \%$ control group had this feeling.

1.4.2. Final Examination Analysis. We used T-test to compare the final examination results between experimental group (No. 35) and control group (No. 31). Statistics showed that the two groups had significant differences $(p=0.049,<0.05)$ (Tables 3 and 4$)$.

It is clear that the final examination score of experimental group was higher than that of control group and the difference was significant $(p=0.049,<0.05)$. It indicated that multidimensional interaction had improved students' learning results.

\section{Discussion}

The above research results show that multidimensional interaction with the assistance of omnimedia has a positive effect on online English learning. In the beginning, students 


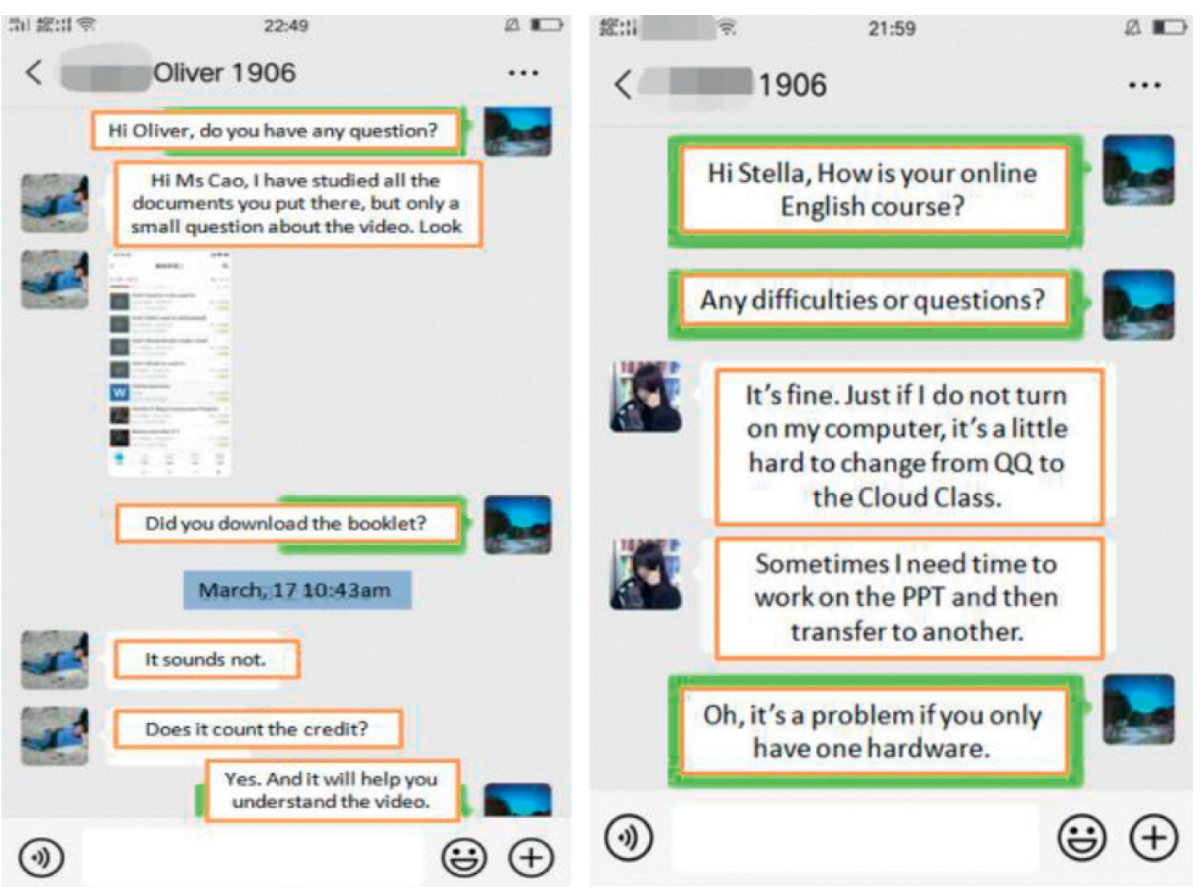

Figure 7: Screenshot of WeChat interaction.

are more concentrated on online English learning as a variety of functions in different platforms have been used. These platforms, which provided words, sounds, pictures, audios, videos, etc., play an important role in drawing students to join various online activities. This increases opportunities for student-teacher and student-student interactions which is comparable to or even exceeded that of offline learning. It also lets the individual students feel they have received more attention. As a result, multidimensional interaction arouses the students' enthusiasm for online learning. It also indicates that most students have a very positive response to the benefits of using online learning with the support of educators [25]. Furthermore, for shy students who tend to be passive, online learning helps them actively answer questions without worrying about other interruptions such as their facial complexion. Overall, shy students tend to speak out in online learning more than offline. The results have coincided with Buelow's et al. research that students just felt that it was a great way for shy students to speak up in online discussion [9]. Besides, online interaction gives students' deep impressions, for many students expose their oral English drawbacks when speaking. They also receive feedback directly from online interaction. When they send messages, they need to review and check the message they have sent, which help them realize their pronunciation drawbacks. Moreover, the final examination results from experimental group and control group have statistically significant differences, meaning that online student engagement has strongly verified the difference of their online learning [11]. For language learning, if students participate in the activities actively, they will have more chances to practice and improve their language competence, whereas those who do not take part in or have limitations to join the multidimensional interactions receive less chances to practice which resulted in lower grades. Although there are significant differences between experimental group and control group in the aspects of questionnaire and final examination, we still need to pay attention to those $30 \%$ experimental students who did not feel any superiority of online learning. This could be explained by the students adhering to their own methods to acquire knowledge. Besides, we should not ignore the fact that technology support is also one of the factors affecting students' online learning. As from previous WeChat conversation (Figure 7), students need to transfer from one platform to the other while learning, which is not quite convenient. As teachers, we need to pay more attention to the students' learning needs and learning process. Making good use of online learning superiority, we will improve students' online learning results.

2.1. Reflection. Multidimensional interaction with the assistance of omnimedia reasonably grasps the attention of students in online English teaching and makes the teaching more effective. In classes where multidimensional interaction has been implemented, students rate online learning as more favourable. Multidimensional interaction has a positive effect on the students' study results. Still, we need to pay attention to the following.

From technical aspect, Internet, hardware, and software are the foundation to support the online learning. Easily accessible human-technology interface will yield twice the result with the half effort. Along with technology development, both teachers and students should make good use of omnimedia network platform, especially the commonly used platforms to communicate interactively to improve teaching and learning results. 


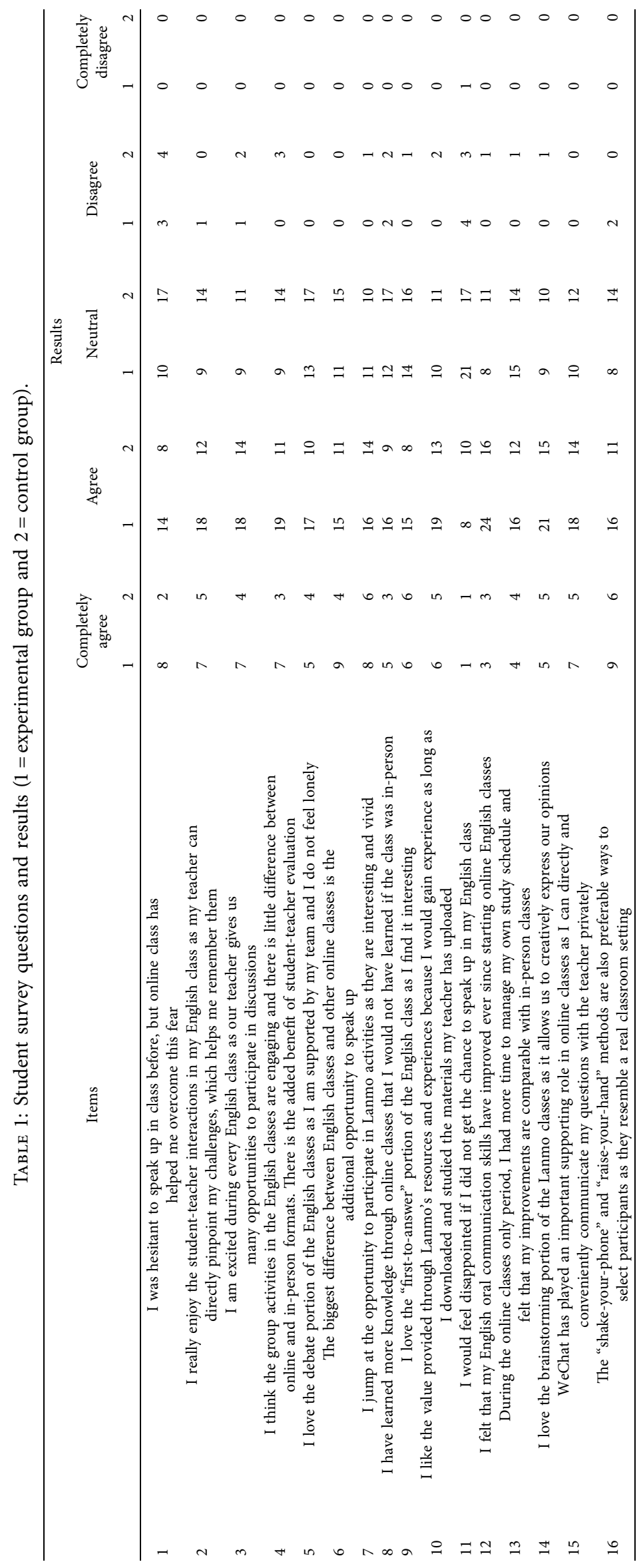


TABLE 2: T-test of experimental group (No. 35) and control group (No. 31).

\begin{tabular}{|c|c|c|c|c|}
\hline Items & Groups & Mean & Std. deviation & Sig. \\
\hline \multirow{2}{*}{ Completely agree and agree } & Experimental group & 22.94 & 4.250 & \multirow{2}{*}{$0.044^{*}$} \\
\hline & Control group & 19.75 & 4.328 & \\
\hline \multirow{2}{*}{ Neutral } & Experimental group & 11.19 & 3.331 & \multirow{2}{*}{$0.000^{* *}$} \\
\hline & Control group & 15.94 & 3.473 & \\
\hline \multirow{2}{*}{ Completely disagree and disagree } & Experimental group & 0.88 & 1.455 & \multirow{2}{*}{0.331} \\
\hline & Control group & 1.88 & 1.408 & \\
\hline
\end{tabular}

${ }^{*} p=0.44,<0.05 ;{ }^{* *} p \leq 0.001$.

TABLE 3: Data of the final examination.

\begin{tabular}{lcccc}
\hline Groups & Numbers & Mean & Std. deviation & Std. error mean \\
\hline Experimental & 35 & 67.40 & 10.094 & 1.706 \\
Control & 31 & 62.68 & 8.852 & 1.590 \\
\hline
\end{tabular}

TABle 4: T-test of the final examination (95\% confidence interval of the difference).

\begin{tabular}{lcccc}
\hline & $\mathrm{t}$ & Sig. & Mean difference & Std. error difference \\
\hline Equal variances assumed & 2.009 & $0.049^{*}$ & 4.723 & 2.351 \\
Equal variances not assumed & 2.025 & 0.047 & 4.723 & 2.332 \\
\hline
\end{tabular}

${ }^{*} p=0.049,<0.05$.

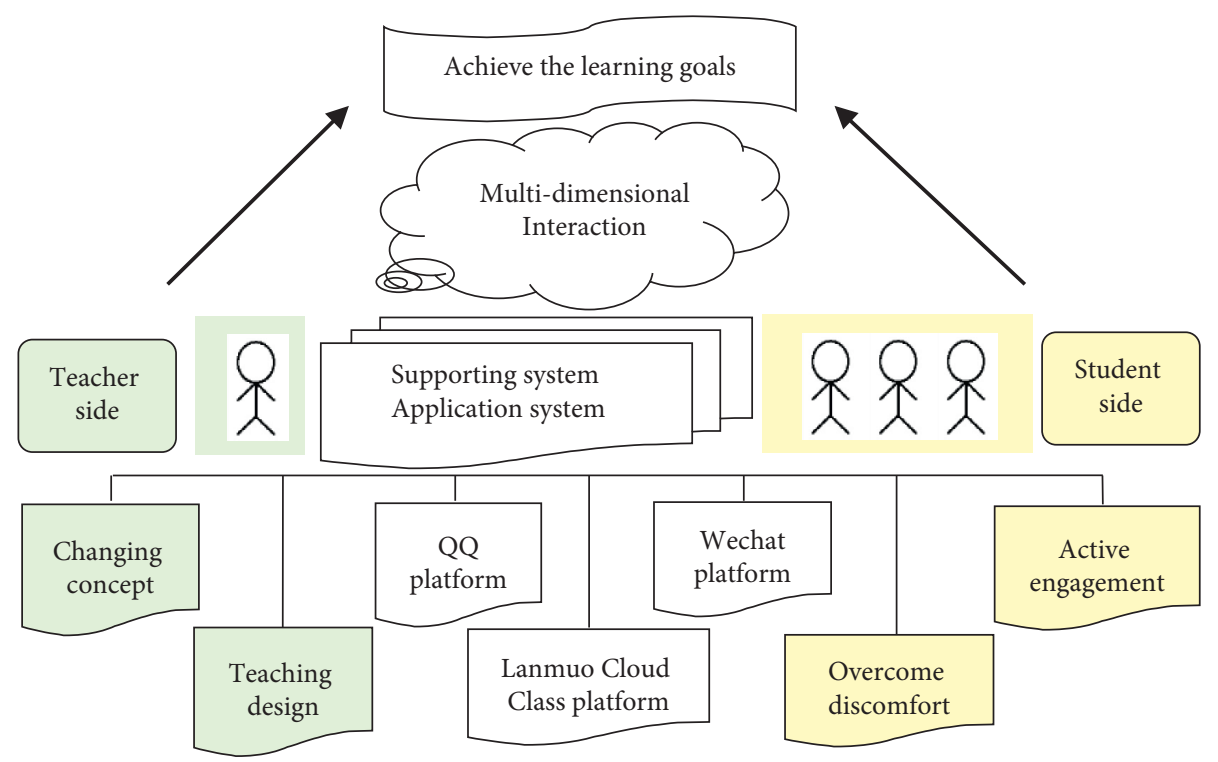

FIGURE 8: Research results.

From teaching aspect, teachers should diligently create teaching plans to implement online interaction more effectively in order to match the effectiveness of offline teaching. Online teaching should not be regarded as a burden. Teaching with personal bias or strictly adhering to conservative traditional teaching methods is not conducive toward effective online teaching.

For students, overcoming the inner discomfort of online learning and actively participating in the process of interactive learning will bring positive improvements to their online learning experience. Investigation shows that as long as students put enough passion into online learning, actively engage in class activities, and take part in the multidimensional interactions with teachers and classmates, they will realize significant gains from online education (Figure 8).

\section{Conclusion}

In summary, studying new technologies and conceptions, changing the outdated ideas and habits, and adapting to reformations in technology and education with open minds ultimately facilitate the embodiment of student-oriented teaching process for both students and teachers. This paper explores the teaching modes of online multidimensional interactive English teaching with the assistance of QQ, 
Lanmo Cloud Class, and WeChat platforms and has shown preliminary results. It helps students to actively engage in online English learning and achieve the satisfactory results. It perhaps opens a window for IT professionals to design more accessible systems for teachers and students and reach the goals of $5 \mathrm{~A}$. In the future, additional resources should be dedicated to studying student learning behaviours and processing and accumulating more experience for integrating technology into online-offline hybrid education.

\section{Data Availability}

Data sharing is not applicable to this article as no datasets were generated or analyzed during the current study.

\section{Conflicts of Interest}

The author declares no potential conflicts of interest with respect to the research, authorship, and/or publication of this article.

\section{Acknowledgments}

This study was supported by the Thirteenth Five-Year Plan of Shaanxi Province Education Science Project of 2020 "Study on the English Learning Process of ApplicationOriented University Students Based on the Paradigm of PAD," Project no. SGH20Y1475.

\section{References}

[1] J. X. Liu, X. X. Wang, and J. Wei, "Effects of blended teaching of English audio-visual \& speaking course in the "Internet Plus" environment---Sino-Us cross-culture EFL blended teaching class with real-time technology," JournalTechnology Enhanced Foreign Language Education, vol. 6, pp. 105-112+10, 2020.

[2] L. Yang, H. J. Zeng, and B. Y. Gao, "Research on the smart learning environment based on cloud computing," Journal Modern Educational Technology, vol. 11, pp. 26-32, 2018.

[3] H. L. Wang, "A probe into the teaching reform of translation major in the era of Cloud Computing," Journal Theory and Practice of Contemporary Education, vol. 6, pp. 61-62, 2016.

[4] X. F. Wang and J. B. Gao, "An English-Chinese Artificial Intelligence dictionary," Shanghai Jiaotong University Press, vol. 9, p. 18, 2019.

[5] S. A. S. insights, "Big Data Insights. Big Data: What it Is and Why it Matters," 2021SAS Institute Inc., 2021, https://www. sas.com/en_us/insights/big-data/what-is-big-data.html.

[6] D. Zou and H. R. Xie, "Vocabulary learning based on learnergenerated pictorial annotations: using Big Data as learning resources," J Sustainability, vol. 13, p. 5767, 2021.

[7] L. Xiao, "Motivation of students autonomous learning in English MOOC teaching based on Big Data analysis," $\mathrm{Ca}$ nadian Journal of Physics: Conference Series, vol. 1881, no. 2, Article ID 022082, 2021.

[8] Y. Zhang, "Research on College English Online learning platform model based on big data technology," Canadian Journal of Physics: Conference Series, vol. 1648, Article ID 042090, 2020.
[9] J. R. Buelow, T. Barry, and L. E. Rich, "Supporting learning engagement with online students," Journal of Online Learning, vol. 4, pp. 313-340, 2018.

[10] C. H. Ding, "Research on the multi-dimensional and interactive SPOC blended teaching model," Journal of Modern Educational Technology, vol. 7, pp. 102-108, 2017.

[11] S. Mehall, "Purposeful interpersonal interaction in online learning: what is it and how is it measured?" Journal of Online Learning, vol. 1, pp. 182-204, 2020.

[12] Y. K. Wang and B. Liu, "A study on the practice of a blended academic English program for postgraduates," Journal of Foreign Languages and Their Teaching, vol. 5, pp. 10-19+147, 2019.

[13] Sociology Editorial Board of Encyclopedia of China, Encyclopedia of China, Encyclopedia of China Publishing House, Beijing, China, 1991.

[14] B. Z. Wang, Q. J. Guo, and D. H. Liu, A Brief History of Education in China, pp. 80-81, Beijing Normal University Publishing Group, Beijing, China, 2008.

[15] G. M. Moore, "Editorial: three types of interaction," American Journal of Distance Education, vol. 2, pp. 1-7, 1989.

[16] A. S. Palinscar and A. L. Brown, "Reciprocal teaching of comprehension-fostering and comprehension-monitoring activities," Journal of Cognition and instruction, vol. 2, pp. 117-175, 1984.

[17] D. Fu, X. Feng, and Y. Wang, "Application of multi-dimensional interactive online teaching method in the course teaching of "Mechatronics System Design and Practice"," Journal of Research in Higher Education of Engineering, vol. 1, pp. 193-196, 2021.

[18] X. Zhou, "The Effect of Multi-dimensional interaction on L2 vocabulary acquisition," Journal of Modern Foreign Languages, vol. 5, pp. 647-660, 2018.

[19] X. Y. Bao and Z. L. Liang, "Research on mobile teaching model based on WeChat platform," Journal of Microcomputer Applications, vol. 12, pp. 73-77, 2019.

[20] J. Zhou and D. Chen, "The exploration of adults online education models in omnimedia environment," Journal of Heilongjiang Researches on Higher Education, vol. 3, pp. 8184, 2015.

[21] Z. T. Zhu and H. C. Peng, "Omnimedia learning ecology: a practical solution to cope with schooling difficulties during a large-scale epidemic," Journal of China Educational Technology, vol. 3, pp. 1-6, 2020.

[22] Ministry of Education of the People's Republic of China, "Statistical Bulletin of the National Education Development in 2018," 2019, https://www.moe.gov.cn/jyb_sjzl/sjzl_fztjgb/ 201907/t20190724_392041.html.

[23] L. Kazanidis, N. Pellas, P. Fotaris, and A. Tsinakos, "Facebook and Moodle integration into instructional media design courses: a comparative analysis of students' learning experiences using the community of inquiry (CoI) model," International Journal of Human-Computer Interaction, vol. 34, no. 10, pp. 932-942, 2018.

[24] H. L. Gao and Y. Y. Qiao, "Online computer course teaching design based on Tencent and Cloud Class platforms," Journal of Computer Education, vol. 11, pp. 58-61, 2020.

[25] M. Maskun, T. Rusman, S. Suroto, and F. Rahmawati, Student perceptions of online learning," International Journal of Multicultural and Multireligious Understanding, vol. 2, no. 7, pp. 67-73, 2020. 